\title{
Experimental validation of a continuous-time MCSI algorithm with bounded adaptive gains
}

\author{
Umberto Montanaro ${ }^{\mathrm{a}, *}$, Ramon Costa-Castellób ${ }^{\mathrm{b}}$ Josep M. Olmc, \\ Ciro Larco Barros ${ }^{\mathrm{d}}$ \\ ${ }^{a}$ Department of Mechanical Engineering Sciences, University of Surrey, Guilford, UK \\ ${ }^{b}$ Department of Automatic Control, Universitat Politècnica de Catalunya, Barcelona, Spain \\ ${ }^{c}$ Department of Mathematics \& Institute of Industrial and Control Engineering, Universitat \\ Politècnica de Catalunya, Barcelona, Spain \\ ${ }^{d}$ Department of Electrical and Electronic Engineering, Universidad de Cuenca, Cuenca, Ecuador
}

\begin{abstract}
Model reference adaptive control algorithms with minimal controller synthesis have proven to be an effective solution to tame the behaviour of linear systems subject to unknown or time-varying parameters, unmodelled dynamics and disturbances. However, a major drawback of the technique is that the adaptive control gains might exhibit an unbounded behaviour when facing bounded disturbances. Recently, a minimal controller synthesis algorithm with an integral part and either parameter projection or $\sigma$-modification strategies was proposed to guarantee boundedness of the adaptive gains. In this article, these controllers are experimentally validated for the first time by using an electro-mechanical system subject to significant rapidly varying disturbances and parametric uncertainty. Experimental results confirm the effectiveness of the modified minimal controller synthesis methods to keep the adaptive control gains bounded while providing, at the same time, tracking performances similar to that of the original algorithm.
\end{abstract}

Keywords: Model reference adaptive control, minimal controller synthesis, parameter projection, $\sigma$-modification, disturbances

\footnotetext{
* Corresponding author: Umberto Montanaro

Email addresses: u.montanaroesurrey . ac.uk (Umberto Montanaro), ramon. costa@upc. edu (Ramon Costa-Castelló), josep. olm@upc . edu (Josep M. Olm), ciro. larco@ucuenca . edu . ec (Ciro Larco Barros)
} 


\section{Introduction}

Since its introduction in the 1950's, adaptive control has been an active field of research, and improvements of adaptive algorithms, including its combinations with other control techniques such as sliding modes, neural networks, iterative learning control, fuzzy control or boundary control, as well as new fields of application for adaptive controllers, are reported in the technical literature on a continuing basis, see for example [35, 38, 2, 19, 27, 17, 16, 39, 6, 20, 18, 37, 15] and references therein. Among these solutions, Model Reference Adaptive Control (MRAC) strategies with Minimal Controller Synthesis (MCS) [29] can steer the dynamics of linear plants in controllable canonical form so as to track those of a pre-selected reference model despite parameter uncertainty, unmodelled nonlinear dynamics and disturbances. Examples of engineering applications where MCS methods have shown to provide excellent tracking performance without any knowledge of plant parameters and disturbances include electronic throttle valves [24], common rail systems [23], electromechanical valve actuators for future camless engines [14], synchronization of chaotic systems [32], and shaking tables [33], just to name a few.

After the seminal papers [29, 30], MCS strategies have been also extended from a theoretical viewpoint with the aim of improving tracking performance or controlling more challenging classes of systems, e.g., discontinuous systems. For example, multi-input continuous-time systems can be controlled via the decentralized MCS [4]; rapidly time-varying disturbances can be suppressed by the Extend MCS (EMCS), which embeds an additional switching action [31]; instead, the Integral MCS (MCSI) [34] includes an integral control action where the integral gain is adaptive too, and it can be used to further improve tracking performance. Recently, MCS control schemes for piecewise affine systems have been proposed $[13,12,10]$. The MCS algorithm for discrete-time systems was presented in [9, 8], and extended to piecewise linear systems in [11]. In the discrete-time framework, integral and switching control actions have been proposed in [23] and [24], respectively.

However, MCS strategies, similarly to other MRAC methods, occasionally show an unbounded drift of the adaptive gains when the plant is subjected to external disturbances or unmodeled dynamics $[8,1]$ that may lead to larger tracking errors or even instability. Such an issue was addressed in [25] for discretetime MCS algorithms while a continuous-time counterpart was recently reported in [26]. Specifically, in [26] two MCS algorithms where proposed. Both algorithms include an integral action and are equipped with parameter projection or a 
$\sigma$-modification strategy; the algorithms are termed as MCSI with Parameter Projection (MCSI-PP) and MCSI with sigma modification ( $\sigma$-MCSI), respectively. Nevertheless, the validation presented in [26] was only numerical, i.e., through computer simulations.

The aim of this paper is to experimentally validate for the first time the MCSI$\mathrm{PP}$ and $\sigma$-MCSI strategies. The experimental validation is carried out by using a roto-magnet system, i.e., an electro-mechanical device consisting of a rotating bar with two magnets at the ends that is actuated by a DC motor and moves within a magnetic field created by two fixed magnets. This plant can be modelled as a linear system where the angular speed of the bar is the output and the voltage applied to the DC motor is the input to the system. The control of the angular speed is challenging as the system is subjected to significant rapidly varying disturbances caused by the magnetic interaction between the bar and the fixed magnets [7]. As shown in [7], a reference angular speed can be imposed to the roto-magnet system through the use of internal model principle-based strategies when the plant parameters are precisely known together with a detailed model for the disturbance acting on the plant and the target rotating speed is kept constant [7]. However, as experimentally proven in [3], closed-loop tracking performance significantly decays in the face of a time-varying reference speed or plant parameter uncertainties, thus making adaptive strategies a possible solution for controlling the roto-magnet system under these working conditions. Specifically, in [3] it was experimentally shown that an MCS adaptive algorithm can maintain the tracking error bounded in any plant working condition also when the model-based controller fails. Nevertheless, an unbounded growth of the MCS adaptive gains was detected which was caused by the disturbance acting on the plant. It is remarked that the unbounded drift of the gains can result also in closed-loop instability for the roto-magnet, thus it must be prevented. For these reasons, the roto-magnet plant is a perfect experimental example to validate the MCSI-PP and $\sigma$-MCSI algorithms.

Experimental results presented in this paper confirm the effectiveness of the novel MCS methods to control the roto-magnet system while keeping the adaptive control gains bounded when compared to a pre-existing MCS solution. Specifically, we show that an unbounded drift of the adaptive gains and closed-loop instability arise when using the classical MCSI in [28]. Then it is shown that both the MCSI-PP and the $\sigma$-MCSI provide similar tracking performance to those given by the classical MCSI algorithm during its stable working regime but with the corresponding adaptive gains bounded, thus experimentally confirming the theoretical findings in [26].

The article is organized as follows. The MCSI control algorithms with bounded 
adaptive gains are presented in Section 2. Then, the experimental setup is described in Section 3. Furthermore, in Section 3 it is also carried out an experimental comparison between a pre-existing adaptive MCS and the internal model-based control strategy for disturbance rejection proposed in [7] with the aim to show the loss of tracking performance of the model-based disturbance rejection solutions in presence of time-varying reference inputs and unknown plant parameters, thus suggesting the use of adaptive MCS strategies for such working conditions. In Section 4, the novel MCS methods presented in [26] are experimentally shown to be effective to bound the adaptive control gains and to prevent closed-loop instability. Finally, conclusions are drawn in Section 5.

In the rest of the paper $\mathbb{R}^{n}$ and $\mathbb{R}^{n \times n}$ denote the $n$-dimensional real space and the set of real matrices of dimension $n \times n$, respectively; $\mathcal{C}$ is the set of continuous scalar functions; $L_{2}$ is the space of functions such that the square of its 2-norm is Lebesgue integrable; $L_{\infty}$ is the space of functions with real essential supremum norm; $(\cdot)^{T}$ denotes the transpose of a vector or matrix; $\mathcal{O}_{n}$ is the null vector in $\mathbb{R}^{n} ;\|\cdot\|$ denotes the 2 -norm of a vector; $\lambda_{\min }(\cdot)$ and $\lambda_{\max }(\cdot)$ are the minimum and maximum eigenvalue of a symmetric square matrix, respectively.

\section{MCSI algorithms with adjusted adaptive mechanisms}

Consider a plant of the form

$$
\dot{x}=A x+B u+B_{e} \delta, \quad x\left(t_{0}\right) \in \mathbb{R}^{n},
$$

where $x \in \mathbb{R}^{n}$ and $u \in \mathbb{R}$ are the state and the input of the system, respectively, with $t_{0} \in \mathbb{R}$ being the initial time instant. The scalar disturbance $\delta=\delta(t)$ acting on the plant dynamics belongs either to $L_{2} \cap L_{\infty}$ or $\mathcal{C} \cap L_{\infty}$, with $\mathcal{C}$ being the set of continuous scalar functions. In addition, the system matrices $A \in \mathbb{R}^{n \times n}, B \in \mathbb{R}^{n}$ are in controllable canonical form, i.e.

$$
A=\left[\begin{array}{ccccc}
0 & 1 & 0 & \ldots & 0 \\
0 & 0 & 1 & \ldots & 0 \\
\vdots & \vdots & \vdots & \ddots & \vdots \\
0 & 0 & 0 & \ldots & 1 \\
a_{1} & a_{2} & \ldots & \ldots & a_{n}
\end{array}\right], B=b B_{e}, \quad B_{e}=\left[\begin{array}{c}
0 \\
0 \\
\vdots \\
1
\end{array}\right]
$$

The plant parameters in (2) are assumed to be constant and unknown, but belonging to known bounded sets, i.e., $a_{i} \in\left[a_{i}^{l}, a_{i}^{u}\right]$, with $a_{i}^{u}>a_{i}^{l}, i=1, \ldots, n$, 
and $b \in\left[b^{l}, b^{u}\right]$ with $b^{u}>b^{l}>0$, where $a_{i}^{l}, a_{i}^{u}, b_{i}^{l}, b_{i}^{u}$ are known constants.

The control objective for the MCSI algorithm is to impose to system (1) the dynamics of a given reference model while keeping all the closed-loop signals bounded.

The reference model is an asymptotically stable LTI system of the form

$$
\dot{x}_{m}=A_{m} x_{m}+B_{m} r, \quad x_{m}\left(t_{0}\right) \in \mathbb{R}^{n}
$$

where $x_{m} \in \mathbb{R}^{n}$ is the reference model state, $A_{m} \in \mathbb{R}^{n \times n}, B_{m} \in \mathbb{R}^{n}$ are the reference model matrices given in the same canonical form as those of the plant, i.e.,

$$
A_{m}=\left[\begin{array}{ccccc}
0 & 1 & 0 & \ldots & 0 \\
0 & 0 & 1 & \ldots & 0 \\
\vdots & \vdots & \vdots & \ddots & \vdots \\
0 & 0 & 0 & \ldots & 1 \\
a_{m, 1} & a_{m, 2} & \ldots & \ldots & a_{m, n}
\end{array}\right], \quad B_{m}=b_{m} B_{e}
$$

with $A_{m}$ Hurwitz and $b_{m} \in \mathbb{R}$, and $r=r(t) \in \mathbb{R}$ is a bounded time-varying function.

The control action provided by both the MCSI-PP and the $\sigma$-MCSI control algorithms can be written as [26]:

$$
u=u_{M C S}+u_{I},
$$

with

$$
\begin{aligned}
u_{M C S} & =K_{X} x+K_{R} r \\
u_{I} & =K_{I} x_{I},
\end{aligned}
$$

where

$$
\dot{x}_{I}=x_{e}+f_{e} \quad \text { and } \quad x_{e}=x_{m}-x ;
$$

the adaptive gains are computed as

$$
\begin{aligned}
K_{X} & =\phi_{X}^{T}+\beta_{X} y_{e} x^{T} \quad \text { and } \quad \dot{\phi}_{X}^{T}=\alpha_{X} y_{e} x^{T}+f_{X}^{T}, \\
K_{R} & =\phi_{R}+\beta_{R} y_{e} r \quad \text { and } \quad \dot{\phi}_{R}=\alpha_{R} y_{e} r+f_{R}, \\
K_{I} & =\phi_{I}^{T}+\beta_{I} y_{e} x_{I}^{T} \quad \text { and } \quad \dot{\phi}_{I}^{T}=\alpha_{I} y_{e} x_{I}^{T}+f_{I}^{T},
\end{aligned}
$$


$\alpha_{X}, \alpha_{R}, \alpha_{I}, \beta_{X}, \beta_{R}$ and $\beta_{I}$ being positive adaptive weights, while

$$
y_{e}=C_{e} x_{e}, \quad C_{e}=B_{e}^{T} P, \text { and } P A_{m}+A_{m}^{T} P=-Q,
$$

with $Q=Q^{T}>0$. Notice that the existence of $P$ is guaranteed by the Hurwitz assumption on the reference model matrix $A_{m}$.

The functions $f_{e}, f_{X}, f_{R}$ and $f_{I}$, which do not appear in classical MCS algorithms, depend on the strategy exploited to bound the evolution of both the integral variable, $x_{I}$, and the adaptive gains, namely, parameter projection or $\sigma$ modification, and will be described in detail in the following subsections. We remark that to design these terms, the only extra assumption that is required with respect to the MCS algorithms available in the technical literature is that lower and upper bounds for the plant parameters are known. The reader is referred to [26] for further details.

The stack of the integral part of the adaptive gains is denoted as $\phi$ :

$$
\phi^{T}=\left[\phi_{1}, \phi_{2}, \ldots, \phi_{2 n+1}\right]=\left[\phi_{X}^{T} \vdots \phi_{R} \vdots \phi_{I}^{T}\right] .
$$

Moreover, the vector $\phi^{\star} \in \mathbb{R}^{2 n+1}$ is defined as

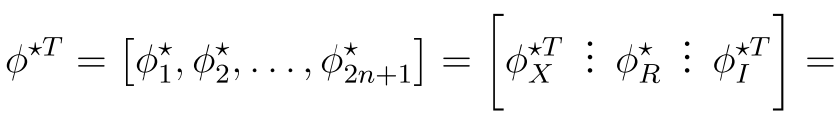

$$
\begin{aligned}
& =\left[b^{-1} B_{e}^{T}\left(A_{m}-A\right) \vdots b^{-1} b_{m} \vdots \mathcal{O}_{n}^{T}\right] \text {, }
\end{aligned}
$$

with

$$
\phi_{X_{j}}^{\star}=b^{-1}\left(a_{m, j}-a_{j}\right), \quad j=1, \ldots, n .
$$

Notice that, as the plant parameters are unknown, the vector $\phi^{\star}$ is unknown. Nevertheless, as the range of variation of the plant parameters is known, one can find two vectors $\phi^{u}, \phi^{l} \in \mathbb{R}^{2 n+1}$, defined as

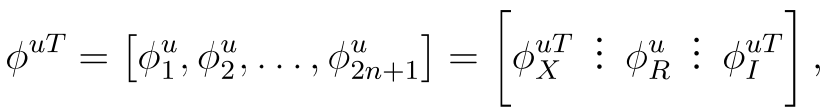

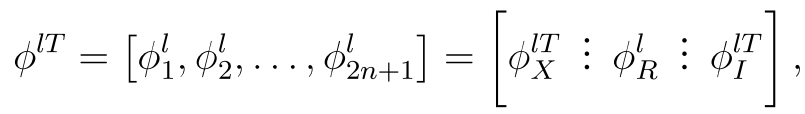


and a positive constant $\mathcal{M}$ such that

$$
\begin{aligned}
\phi^{\star} \in \Gamma_{\phi} & :=\left\{\phi \in \mathbb{R}^{2 n+1}:\|\phi\| \leq \mathcal{M}\right\} \\
\phi^{\star} \in \Lambda_{\phi} & :=\left[\phi_{1}^{l}, \phi_{1}^{u}\right] \times \cdots \times\left[\phi_{2 n+1}^{l}, \phi_{2 n+1}^{u}\right] .
\end{aligned}
$$

Remark 1. The assumption of a preliminary knowledge of a set containing the plant parameters is not as restrictive as it might appear at first: indeed, nominal values for the plant parameters, as well as their range of variation, are often available in engineering problems. In addition, there is no loss of generality in assuming $b^{u}>b^{l}>0$ because if $0>b^{l}>b^{u}$ then the sign of the adaptive gain weights should be accordingly selected. Hence, the requirement is, in fact, that $b$ has a definite sign.

\subsection{MCSI with parameter projection}

Parameter projection proposes a design for the terms $f_{X}, f_{R}$ and $f_{I}$ in (8) that keeps the evolution of $\phi$ within $\Lambda_{\phi}$. This is achieved componentwise as follows:

$$
\begin{aligned}
& f_{X_{j}}=\left\{\begin{array}{c}
0 \text { if } \phi_{X_{j}} \in\left(\phi_{X_{j}}^{l}, \phi_{X_{j}}^{u}\right), \text { or } \phi_{X_{j}}=\phi_{X_{j}}^{l} \text { and } h_{X_{j}} \geq 0, \\
\text { or } \phi_{X_{j}}=\phi_{X_{j}}^{u} \text { and } h_{X_{j}} \leq 0, \\
-h_{X_{j}} \text { otherwise },
\end{array}\right. \\
& f_{R}=\left\{\begin{array}{c}
0 \text { if } \phi_{R} \in\left(\phi_{R}^{l}, \phi_{R}^{u}\right), \text { or } \phi_{R}=\phi_{R}^{l} \text { and } h_{R} \geq 0, \\
\text { or } \phi_{R}=\phi_{R}^{u} \text { and } h_{R} \leq 0, \\
-h_{R} \text { otherwise, }
\end{array}\right. \\
& f_{I_{j}}=\left\{\begin{array}{c}
0 \text { if } \phi_{I_{j}} \in\left(\phi_{I_{j}}^{l}, \phi_{I_{j}}^{u}\right), \text { or } \phi_{I_{j}}=\phi_{I_{j}}^{l} \text { and } h_{I_{j}} \geq 0, \\
\text { or } \phi_{I_{j}}=\phi_{I_{j}}^{u} \text { and } h_{I_{j}} \leq 0, \\
-h_{I_{j}} \text { otherwise, }
\end{array}\right.
\end{aligned}
$$

with

$$
\begin{aligned}
& h_{X_{j}}=\alpha_{X} y_{e} x_{j}, \quad j=1 \ldots n, \\
& h_{R}=\alpha_{R} y_{e} r, \\
& h_{I_{j}}=\alpha_{I} y_{e} x_{I_{j}}, j=1 \ldots n .
\end{aligned}
$$

Notice that, by denoting as

$$
f^{T}=\left[f_{1}, f_{2}, \ldots, f_{2 n+1}\right]=\left[f_{X}^{T} \vdots f_{R} \vdots f_{I}^{T}\right],
$$

according to (15) the generic function $f_{j}$ is zero when the corresponding integral gain $\phi_{j}$ is within boundaries, and it is activated when $\phi_{j}$ reaches any of its boundaries with a tendency to leave the region. In this case, the parameter projection 
term saturates $\phi_{j}$ at the corresponding lower or upper admissible interval value.

Furthermore, a parameter projection-based locking strategy for the integral variable $x_{I}$ is proposed to keep its evolution constrained in a predetermined region of the phase plane. Let this domain be defined as

$$
\Lambda_{I}:=\left[x_{I_{1}}^{l}, x_{I_{1}}^{u}\right] \times \cdots \times\left[x_{I_{n}}^{l}, x_{I_{n}}^{u}\right],
$$

with $x_{I j}^{l}<0$ and $x_{I j}^{u}>0$, for all $j=1, \ldots, n$. Recalling the definition of $x_{I}$ in (7), its confinement within $\Lambda_{I}$ is achieved through the following scheme:

$$
f_{e_{j}}(t)=\left\{\begin{array}{l}
0 \text { if } x_{I_{j}} \in\left(x_{I_{j}}^{l}, x_{I_{j}}^{u}\right), \text { or } x_{I_{j}}=x_{I_{j}}^{l} \text { and } x_{e_{j}} \geq 0 \\
\quad \text { or } x_{I_{j}}=x_{I_{j}}^{u} \text { and } x_{e_{j}} \leq 0 \\
-x_{e_{j}}(t) \text { otherwise. }
\end{array}\right.
$$

In [26] it has been proven that when the parameter-projection in (15)-(18) completes the control action (5), if the disturbance $\delta(t)$ in (1) belongs to $L_{2} \cap L_{\infty}$, then all the closed-loop signals are bounded and, in particular, $\left(\phi, x_{I}\right) \in \Lambda_{\phi} \times \Lambda_{I}$, while $x_{e} \rightarrow 0$ as $t \rightarrow+\infty$. The reader is referred to [26] for the proof, which is here omitted for the sake of brevity.

\subsection{MCSI with $\sigma$-modification}

With $\sigma$-modification, the MCSI gains in (8) are completed by computing $f_{X}$, $f_{R}$ and $f_{I}$ in (8) as

$$
\begin{aligned}
& f_{X}^{T}=-\rho_{X} \sigma_{\phi}(\|\phi\|) \phi_{X}^{T}, \\
& f_{R}=-\rho_{R} \sigma_{\phi}(\|\phi\|) \phi_{R}, \\
& f_{I}^{T}=-\rho_{I} \sigma_{\phi}(\|\phi\|) \phi_{I}^{T},
\end{aligned}
$$

where $\rho_{X}, \rho_{R}$, and $\rho_{I}$ are positive constants. The $\sigma$-function is defined as

$$
\sigma_{\phi}(\|\phi\|)=\left\{\begin{array}{l}
0 \quad \text { if }\|\phi\| \leq \widehat{\mathcal{M}}_{\phi}, \\
\eta_{\phi}\left(\frac{\|\phi\|}{\widehat{\mathcal{M}}_{\phi}}-1\right) \quad \text { if } \widehat{\mathcal{M}}_{\phi}<\|\phi\| \leq 2 \widehat{\mathcal{M}}_{\phi}, \\
\eta_{\phi} \quad \text { if }\|\phi\|>2 \widehat{\mathcal{M}}_{\phi},
\end{array}\right.
$$

with

$$
\widehat{\mathcal{M}}_{\phi}=\sqrt{\frac{\lambda_{\max }\left(\Gamma_{\alpha}^{-1} \Gamma_{\rho}\right)}{\lambda_{\min }\left(\Gamma_{\alpha}^{-1} \Gamma_{\rho}\right)}} \mathcal{M}
$$


while

$$
\begin{aligned}
& \Gamma_{\alpha}=\operatorname{diag}\left(\widehat{\alpha}_{1}, \widehat{\alpha}_{2}, \ldots, \widehat{\alpha}_{2 n+1}\right), \\
& \Gamma_{\rho}=\operatorname{diag}\left(\widehat{\rho}_{1}, \widehat{\rho}_{2}, \ldots, \widehat{\rho}_{2 n+1}\right),
\end{aligned}
$$

where

$$
\begin{aligned}
& \left(\widehat{\alpha}_{i}, \widehat{\rho}_{i}\right)=\left(\alpha_{X}, \rho_{X}\right), i=1, \ldots, n, \\
& \left(\widehat{\alpha}_{n+1}, \widehat{\rho}_{n+1}\right)=\left(\alpha_{R}, \rho_{R}\right), \\
& \left(\widehat{\alpha}_{i}, \widehat{\rho}_{i}\right)=\left(\alpha_{I}, \rho_{I}\right), \quad i=n+2, \ldots, 2 n+1,
\end{aligned}
$$

and $\eta_{\phi}$ is a positive constant satisfying

$$
b \eta_{\phi} \lambda_{\min }\left(\Gamma_{\alpha}^{-1} \Gamma_{\rho}\right)>\frac{3}{4} \lambda_{\min }(Q) .
$$

In addition, the locking strategy for $x_{I}$ is devised in the following $\sigma$-modification fashion

$$
f_{e}^{T}=-\rho_{e} \sigma_{I}\left(\left\|x_{I}\right\|\right) x_{I}^{T}
$$

where $\rho_{e}$ is a positive constant, and $\sigma_{I}(\cdot)$ is defined as

$$
\sigma_{I}\left(\left\|x_{I}\right\|\right)=\left\{\begin{array}{l}
0 \text { if }\left\|x_{I}\right\| \leq \widehat{\mathcal{M}}_{I}, \\
\eta_{I}\left(\frac{\left\|x_{I}\right\|}{\widehat{\mathcal{M}}_{I}}-1\right) \quad \text { if } \widehat{\mathcal{M}}_{I}<\left\|x_{I}\right\| \leq 2 \widehat{\mathcal{M}}_{I}, \\
\eta_{I} \quad \text { if }\left\|x_{I}\right\|>2 \widehat{\mathcal{M}}_{I},
\end{array}\right.
$$

with $\eta_{I}$ and $\widehat{\mathcal{M}}_{I}$ being positive constants.

By defining $\widetilde{x}_{e}^{T}=\left[\begin{array}{ll}x_{e}^{T} & \phi_{e}^{T}\end{array}\right]$, with $\phi_{e}=\phi^{\star}-\phi$, it has been proven in [26] that when the disturbance $\delta(t)$ in (1) is continuous and bounded (i.e., $\delta \in \mathcal{C} \cap L_{\infty}$ ), and the adaptive gains (8) of the control action (5) are completed by using (19) and (23), then, all the closed-loop signals are bounded and, in particular, $\widetilde{x}_{e}$ is globally uniformly ultimately bounded [21]. The latter means that there exists $T \in \mathbb{R}^{+}$and a $\mathcal{K} \mathcal{L}$-class function $\xi: \mathbb{R}^{+} \times \mathbb{R}^{+} \rightarrow \mathbb{R}^{+}$such that $\left\|\widetilde{x}_{e}(t)\right\| \leq \xi\left(\left\|\widetilde{x}_{e}\left(t_{0}\right)\right\|, t-t_{0}\right)$, $\forall t \in\left[t_{0}, t_{0}+T\right)$, and $\left\|\widetilde{x}_{e}(t)\right\| \leq \widehat{\mu}, \forall t \in\left[t_{0}+T,+\infty\right)$, where the ultimate bound $\widehat{\mu}$ can be explicitly computed as a function of the $L_{\infty}$ norm of $\delta(t)$. The reader is referred to [26] for the proof and the computation of $\widehat{\mu}$, which are here omitted again for the sake of brevity.

\section{The roto-magnet system}

The experimental tests have been carried out using an electro-mechanical device known as roto-magnet. This system is composed of a DC motor with a bar 


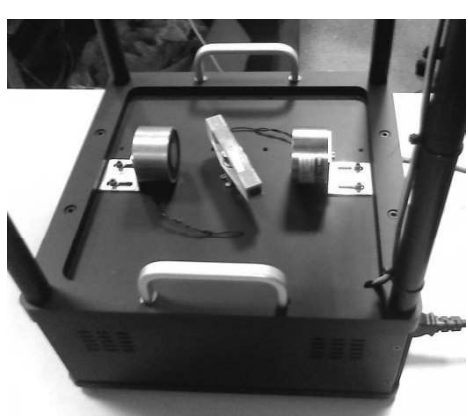

(a)

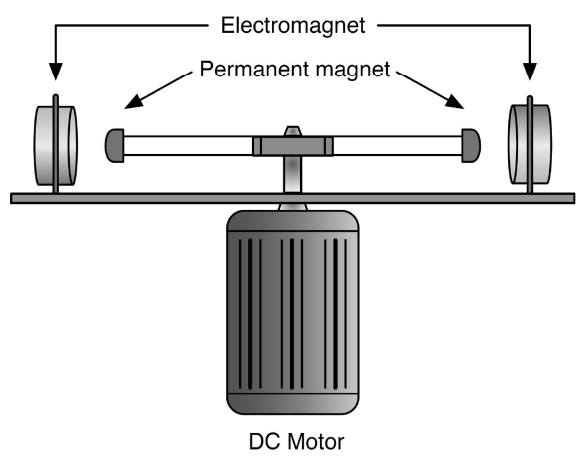

(b)

Figure 1: The roto-magnet system, (a) plant and (b) schematic representation.

attached to its axis in a perpendicular fashion, and with two permanent magnets fixed in both ends. The magnetic field of these magnets is affected by that of two external electromagnets, as shown in Figure 1, thus creating a periodic disturbance for the turning speed. Further details are available in [7].

The system model relating the input voltage to the DC motor, $u$, with the motor axis turning speed, $x$, has been obtained from the unit step response of the plant without disturbances, i.e., without electromagnets, through a parametric identification process. The corresponding expression is that of a first order model and reads as:

$$
\dot{x}=-2.188 x+35.343 u+\delta(t),
$$

where the units of $x$ are revolutions per second. Notice that $\delta$ stands for the disturbance, and is such that $\delta \in \mathcal{C} \cap L_{\infty}$.

The goal for this plant is to keep the speed $x$ regulated at a constant level, $\bar{x}$. When this happens, the pulsating torque created by the electromagnets becomes a periodic disturbance with period $T=\bar{x}^{-1} \mathrm{~s}$.

Remark 2. Internal model-based strategies, such as repetitive control [7], can be used to steer the dynamics of the roto-magnet system when an accurate model for predicting the periodic disturbance acting on system (25) is available and the reference angular velocity is constant. However, tracking performance of the model-based strategy may dramatically decay because of: (i) time-varying operating conditions, i.e., a change in the reference angular speed, or (ii) parameter uncertainty. Instead, adaptive control strategies, such as MCS solutions, provide good tracking performances that depend neither on the plant working conditions 


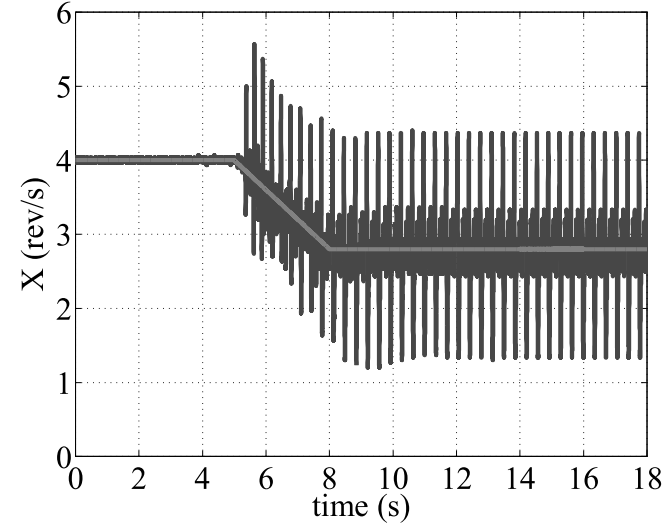

(a)

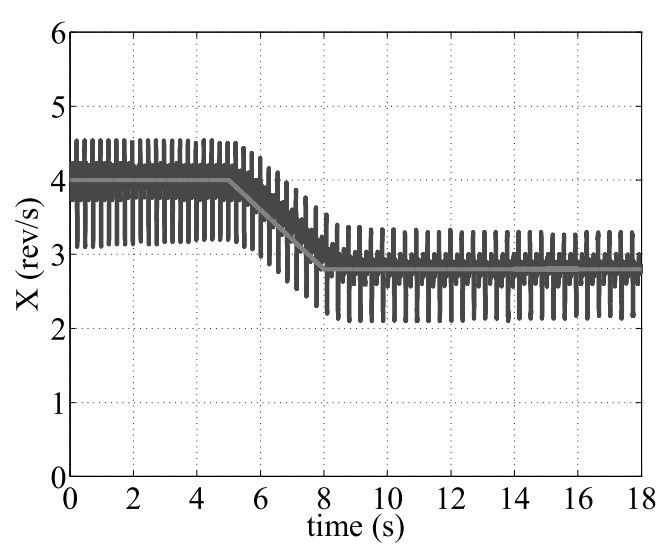

(b)

Figure 2: Tracking performance for time-varying reference speed, speed of the roto-magnet system (blue line), reference speed (red line) in the case of (a) model-based control and (b) MCS.

nor on the knowledge of the plant parameters. Furthermore, MCS strategies do not require a model for estimating the disturbance, thus simplifying the control design. As an example, Figure 2 depicts the experimental plant output for a repetitive controller designed to operate with a reference speed of $\omega=4 \mathrm{rev} / \mathrm{s}$ and a classical MCS in presence of changes in the reference speed. It is noted that the repetitive controller provides better performance up to $5 s$, i.e., as far as the reference speed is that used for the tuning of the model of the disturbance acting on system (25), but it is outperformed by the MCS algorithm when the reference does not match this value. However, the adaptive control gains of the MCS algorithm diverge for a longer experiment as revealed in Figure 3. Additional details about the closed-loop response of the roto-magnet system for time-varying plant working conditions have been recently presented in [3]. Furthermore, in [3] a similar tracking performance decay of the model-based strategy presented in [7], and the performance recovery through the use of an adaptive MCS approach but with an unbounded growth of the adaptive gains were also noted in the case of variations of the plant gain, thus confirming that the roto-magnet system is as an effective benchmark for experimentally evaluating for the first time the novel MCSI-PP and the $\sigma$-MCSI in [26] that guarantee boundedness of the adaptive control gains. 


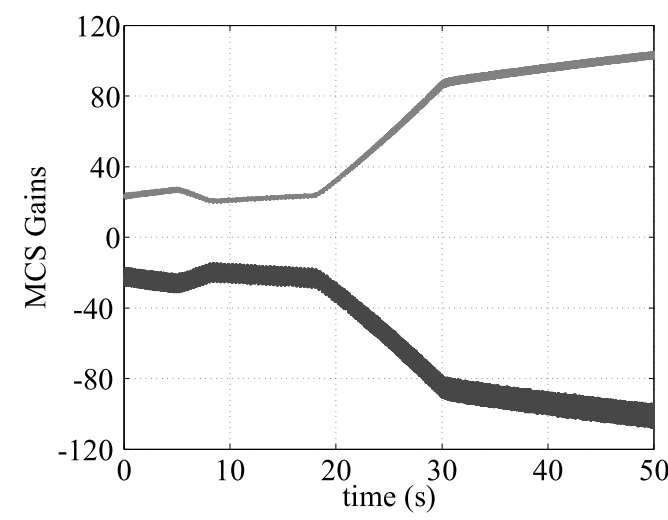

Figure 3: MCS control gains, $K_{X}$ (blue line) and $K_{R}$ (red line) under under a time-varying speed reference.

\section{Experimental results}

In this section, we experimentally investigate the performance of the MCSI adaptive algorithms with bounded adaptive gains introduced in Section 2 on the roto-magnet system described in Section 3. In addition, we compare the closedloop dynamics provided by the MCSI-PP and the $\sigma$-MCSI with respect to those given by the MCSI originally presented in [28].

The selected reference model (3) is a first order, LTI system with $A_{m}=-2$, $B_{m}=2$, and $r=3 \mathrm{rev} / \mathrm{s}$. Hence, the reference model asymptotically stabilizes at $x_{m}^{*}=3 \mathrm{rev} / \mathrm{s}$ with a settling time of $2 \mathrm{~s}$, this being the reference speed, $\bar{x}$, for the plant. Each experiment lasts $90 \mathrm{~s}$.

In order to implement the strategies in Section 2, the output is made coincident with the plant state, i.e. $y=x$, this yielding $P=1$ and $Q=-2 A_{m}=4$ in (9). Furthermore, we have set $\alpha_{X}=\alpha_{R}=\alpha_{I}=5, \beta_{X}=\beta_{R}=\beta_{I}=0.5$. Finally, the integral part of the control gains in (8) is initialized at zero, i.e., $\phi_{X}(0)=0$, $\phi_{R}(0)=0, \phi_{I}(0)=0$. The control system has been implemented via MATLAB/Simulink (R2009b): the discretization uses the ode3 (Bogacki-Shampine) real-time solver with a step size of $1 \mathrm{~ms}$, and it has been coded using an XPC Target 4.2 running over an Intel(R) Core(TM) 2 Quad CPU Q9400 (2.66 GHz) platform. A sampling time of $T_{s}=0.001 \mathrm{~s}$ has been chosen for the DA and AD converters.

Regarding the possible plant parameter variation and in order to satisfy (14), when the MCSI-PP is adopted the integral parts of the adaptive gains have been limited as follows: $\phi_{X}, \phi_{R}, \phi_{I} \in[-0.5,0.5]$. Finally, for the projection of the 
integral variable we have set $x_{I} \in[-0.5,0.5]$ in (18).

Instead, for the $\sigma$-MCSI we use $\mathcal{M}=0.4$ in (14) and $\rho_{X}=\rho_{R}=\rho_{I}=$ 10 in (21), this yielding $\widehat{\mathcal{M}}_{\phi}=0.4$ as well. Moreover, $\eta_{\phi}=10$ in (20), thus guaranteeing the fulfillment of (22). In addition, for the $\sigma$-based locking of the integral variable we have set $\rho_{e}=10$ in (23), while $\eta_{I}=10$ and $\widehat{\mathcal{M}}_{I}=0.1$ in (24).

Remark 3. The adaptive weights in (8) are selected as a trade-off between convergence time and reactivity of the control actions. The coefficients $\alpha_{\zeta}, \zeta \in\{X, R, I\}$ give the rate of variations of the integral part of the adaptive gains. Larger values of $\alpha_{\zeta}$ imply a faster transient of the adaptive gains and can be used to control plants with time-varying parameters [26]. However, if a method for locking the integral part of the adaptive gains is not used, larger values of $\alpha_{\zeta}$ can increase the rate of growth of the control gains in the face of disturbances, which may result in closed-loop instability when the control system is discretised. The coefficients $\beta_{\zeta}$, $\zeta \in\{X, R, I\}$ modulate the magnitude of the proportional part of the adaptive control gains which ideally are zero in the case of perfect tracking of the reference model. Furthermore, in accordance with [22], the use of the proportional part of the adaptive control gains should improve the tracking performance during transients. The coefficients $\rho_{\zeta}$ and $\eta_{\zeta}$, with $\zeta \in\{X, R, I\}$, modulate the rate of discharge of the integral part of the adaptive gains, and therefore the time interval where the norm of the control gains is above the threshold $\widehat{\mathcal{M}}_{\phi}$ when the $\sigma$-modification strategy is used to bound their growth.

For the working conditions described above, Figure 4 shows that the control gains of the pre-existing MCSI algorithm available in the technical literature [28] diverge over time due to the presence of the disturbance in (25), unmodelled dynamics, and the effect of the discretization of the control law [5]. Specifically, Figure 4a shows that $\phi_{X}$ and $\phi_{R}$ diverge linearly over the entire control horizon. Furthermore, the rate of divergence has a different rate of growth over the manoeuvre, i.e., a slow drifting before $t_{c}=43.6 \mathrm{~s}$ and then a more severe divergence rate after this critical time instant. In addition, the gain $\phi_{I}$ does not diverge in the time range $\left[0, t_{c}\right]$, although it shows a quadratic-like growing for time instants larger that $t_{c}$. We remark that in the case of the MCSI the experiment was interrupted around $52 \mathrm{~s}$ in order to prevent damage to the experimental plant and equipment.

The effectiveness of the MCSI-PP and $\sigma$-MCSI to keep the integral part of their adaptive gains bounded over the entire manoeuvre is experimentally confirmed in Figure 5 and Figure 6, respectively. In particular, in Figure 5 it is shown 


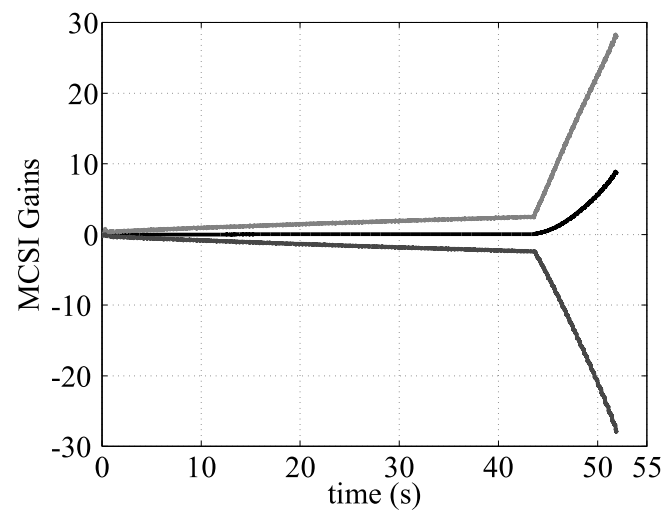

(a)

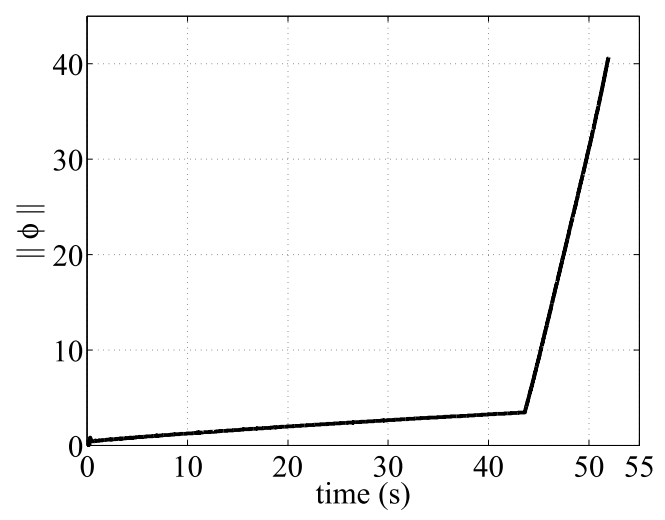

(b)

Figure 4: Integral part of the adaptive gains of the MCSI control algorithm: (a) $\phi_{X}$ (blue line), $\phi_{R}$ (red line) and $\phi_{I}$ (black line); (b) norm $\|\phi\|$.

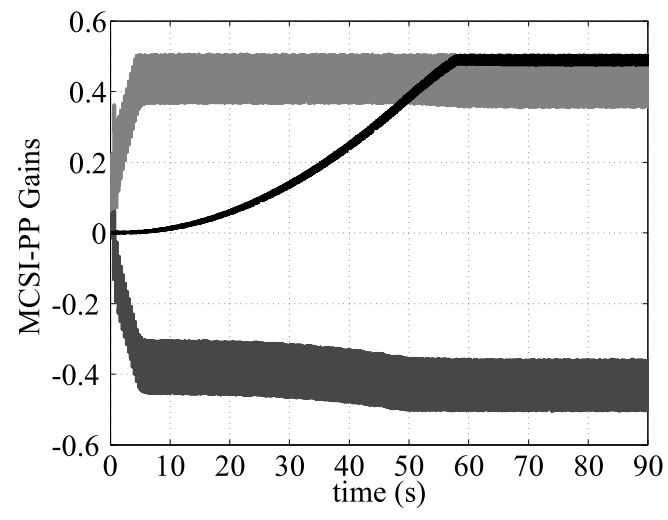

(a)

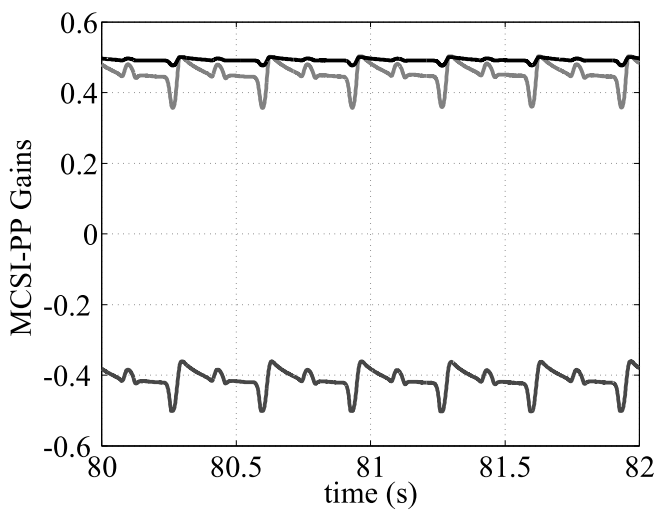

(b)

Figure 5: Integral part of the adaptive gains of the MCSI-PP control algorithm: (a) $\phi_{X}$ (blue line), $\phi_{R}$ (red line) and $\phi_{I}$ (black line); (b) zoom.

that the integral part of the adaptive gains of the MCSI-PP algorithm are confined within the demanded set $\Lambda_{\phi}:=[-0.5,0.5]^{3}$, computed as in (14) from the previously indicated bounds for $\phi_{X}, \phi_{R}, \phi_{I}$. The boundedness of the integral part of the adaptive gains of the MCSI-PP and the $\sigma$-MCSI algorithms is further confirmed in Figure 7, where the norm vector $\phi$ defined in (10) is depicted for both strategies. Specifically, Figure $7 \mathrm{~b}$ shows that in the case of the $\sigma$-MCSI algorithm the 


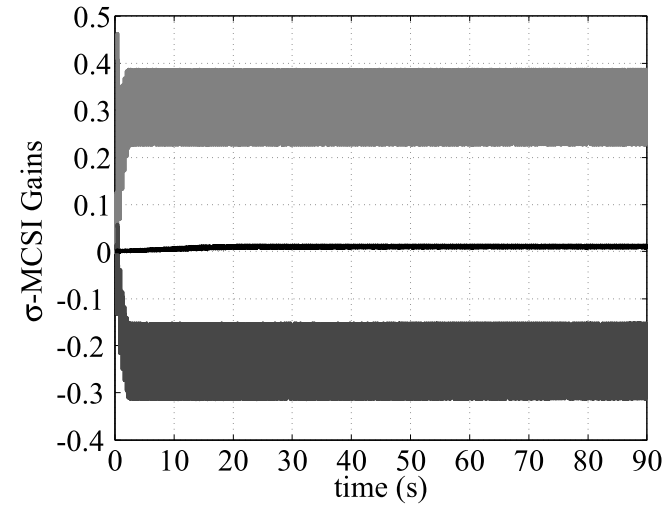

(a)

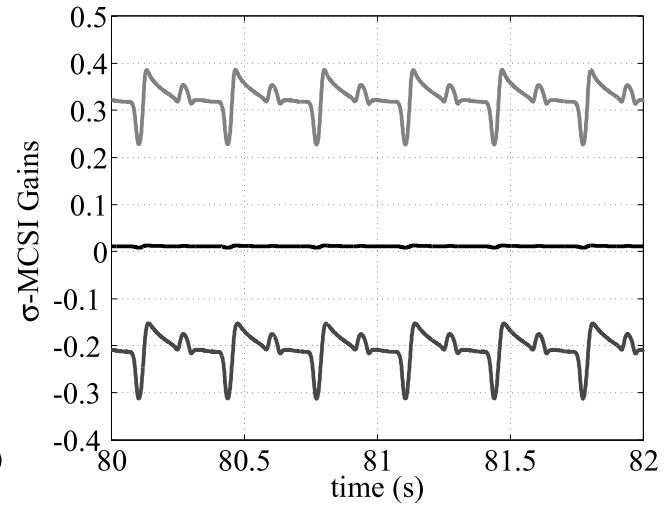

(b)

Figure 6: Integral part of the adaptive gains of the $\sigma$-MCSI control algorithm: (a) $\phi_{X}$ (blue line), $\phi_{R}$ (red line) and $\phi_{I}$ (black line); (b) zoom.

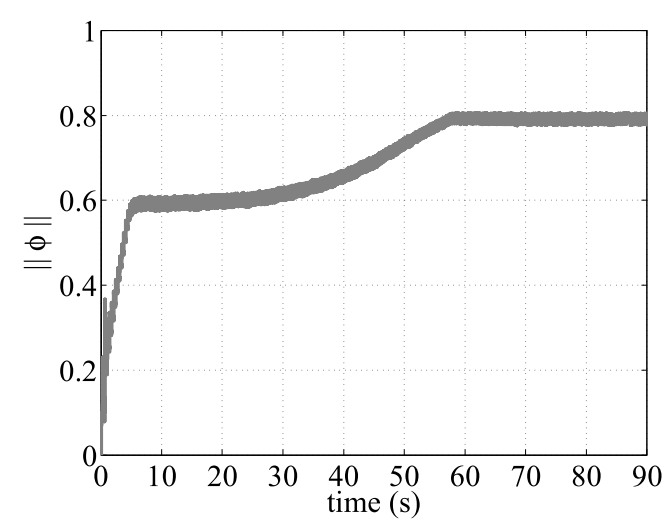

(a)

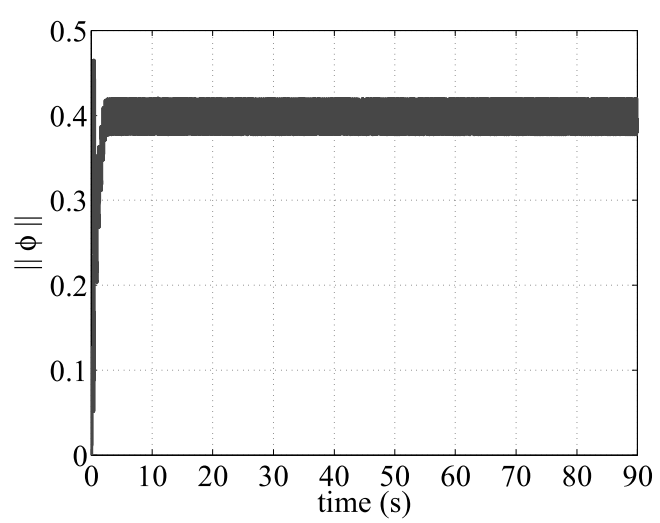

(b)

Figure 7: Norm of the integral part of the adaptive gains $\phi$ of the strategies in Section 2: (a) MCSI-PP; (b) $\sigma$-MCSI.

steady-state value for this norm is around $\widehat{\mathcal{M}}_{\phi}$, which amounts to 0.4 .

It is worth remarking that the MCSI-PP strategy is intended to bound the adaptive gains in the face of $L_{2} \cap L_{\infty}$ disturbances, and the actual perturbation does not belong to this set but to $\mathcal{C} \cap L_{\infty}$, as indicated in Section 3. However, even in this situation, the algorithm is able to effectively lock the control gains.

The closed-loop behaviour in terms of the tracking of the reference profile and 


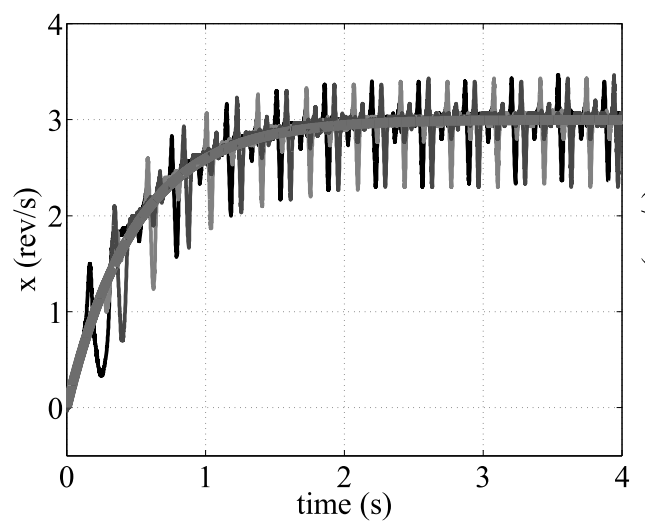

(a)

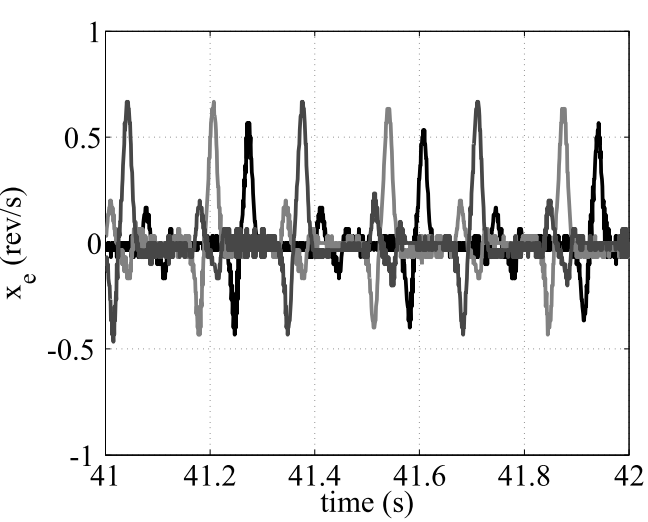

(b)

Figure 8: Closed-loop performance before the critical time instant $t_{c}=43.6 \mathrm{~s}$; MCSI (black line), MCSI-PP (red line) and $\sigma$-MCSI (blue line): (a) transient dynamics at the control activation: reference trajectory (green line); (b) steady-state tracking error.

amplitude of the control action can be analysed for MCSI strategies before and after the critical point (i.e., $t_{c}=43.6$ ).

Specifically, Figure 8 a shows that, at the control activation, the tracking performance provided by both the MCSI-PP and the $\sigma$-MCSI towards the set point are similar to that given by the MCSI algorithm in [28]. Furthermore, also the steady-state tracking error provided by the MCSI-PP and the $\sigma$-MCSI algorithms is comparable to that obtained when the pre-existing MCSI is employed (see Figure $8 \mathrm{~b}$ ). Precisely, in the steady-state, the mean square error with respect to the set-point is $4.5 \%$ for the MCSI, while for the MCSI-PP and the $\sigma$-MCSI this error is $5.2 \%$ and $5.3 \%$, respectively ${ }^{1}$.

A similar comparison can be made also for the control action required to steer the closed-loop dynamics to the reference ones which is shown in Figure 9 for the sake of completeness.

Consequently, the locking strategies introduced in Section 2 do not make closed-loop performance worse than the already available MCSI algorithm. Nevertheless, the remarkable point is that they do not induce a slow drifting of the adaptive gains, as happens with the standard solution (see Figure 4 before the

\footnotetext{
${ }^{1}$ These mean square errors had been computed considering $t \in[20,40] \mathrm{s}$, thus before the critical time instate $t_{c}$.
} 


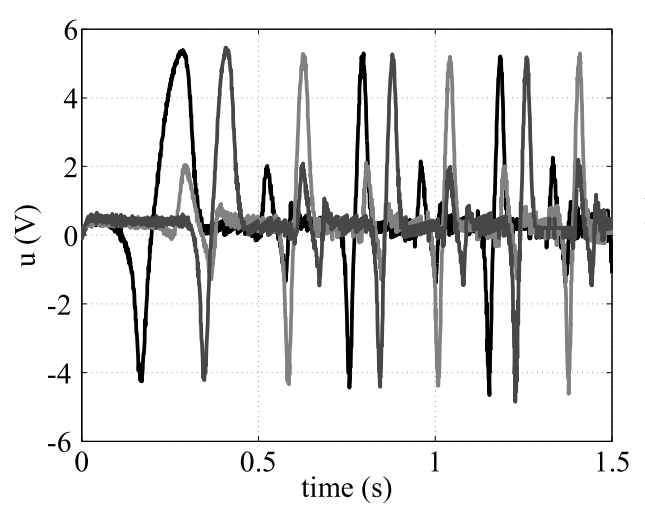

(a)

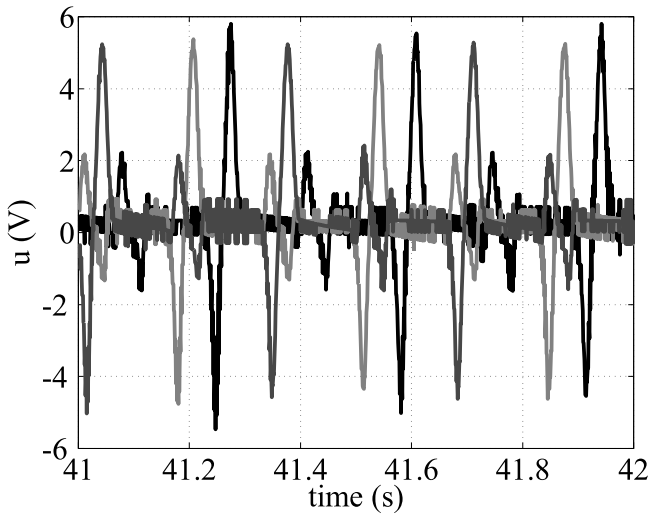

(b)

Figure 9: Control action before the critical time instant $t_{c}=43.6 \mathrm{~s}$; MCSI (black line), MCSI-PP (red line) and $\sigma$-MCSI (blue line): (a) during transient dynamics; (b) in steady state regime.

time instant $t_{c}=43.6 \mathrm{~s}$ ).

For $t>t_{c}$ the increase of the growth rate of the adaptive gains of the MCSI strategy (see Figure 4), severely jeopardizes closed-loop tracking performance, as clearly shown in Figure 10. Specifically, both the magnitude and the frequency of the tracking error increase suddenly, as depicted in Figure 10a and Figure 10b, respectively. Precisely, for the pre-existing MCSI, the mean square error with respect to the set-point shifts from $4.5 \%$ to $36.7 \%$, i.e., there is an increase of more than 8 times of the average tracking error. Instead, the tracking performance provided by the MCSI-PP and $\sigma$-MCSI algorithms are comparable to those obtained for $t<t_{c}$, having a mean square error of $5.16 \%$ and $5.32 \%$ for the MCSI-PP and the $\sigma$-MCSI strategies, respectively ${ }^{2}$.

For $t>t_{c}$, an increase of the amplitude and frequency has been detected also in the control action provided by the pre-existing MCSI algorithm, as shown in Figure 11. Notice that, because of this sudden increase of the magnitude and frequency of the control action, the experiment was interrupted before the originally scheduled time with the aim of preventing damages to the experimental setup.

In addition, Figure 11 reveals that the control actions provided by the MCSI$\mathrm{PP}$ and the $\sigma$-MCSI remain bounded and with an acceptable frequency.

\footnotetext{
${ }^{2}$ These mean square errors have been computed considering $t \in[44,51.5] \mathrm{s}$, thus after the critical time instate $t_{c}$.
} 


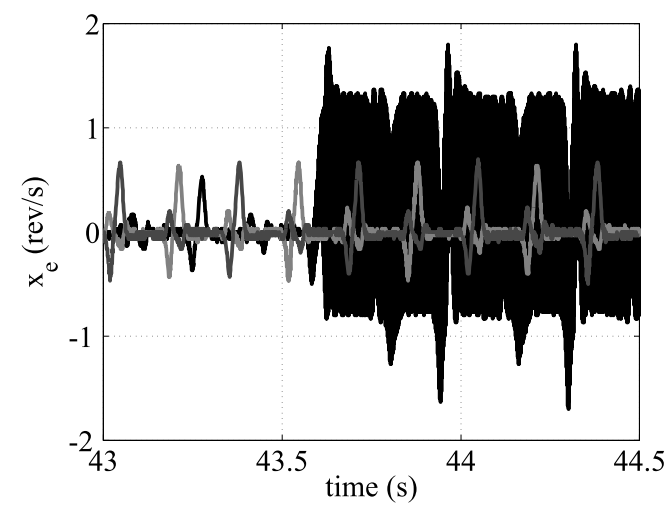

(a)

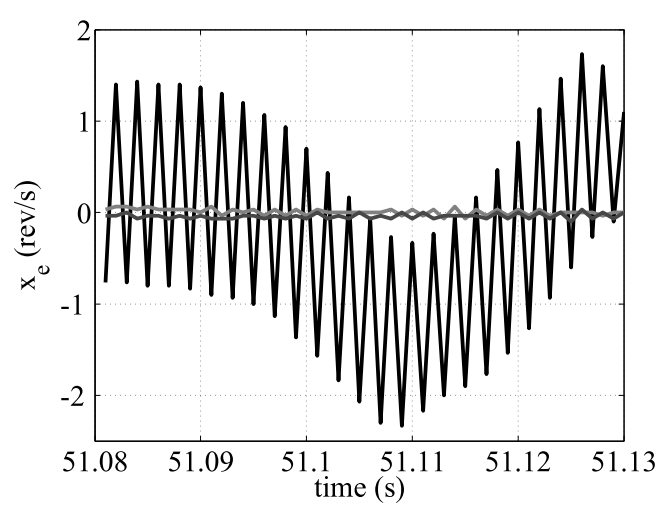

(b)

Figure 10: Closed-loop steady-state tracking error after the critical time instant $t_{c}=43.6 \mathrm{~s}$; MCSI (black line), MCSI-PP (red line) and $\sigma$-MCSI (blue line): (a) $t \in\left[\begin{array}{ll}43, & 44.5\end{array}\right] \mathrm{s}$; (b) $t \in$ $[51.08,51.13] \mathrm{s}$.

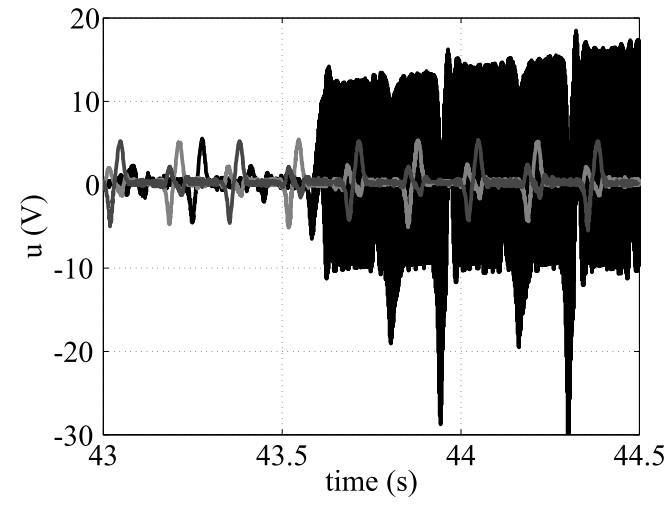

(a)

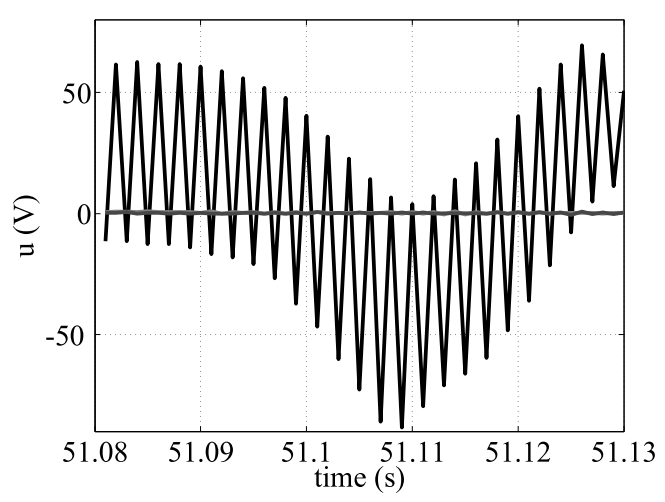

(b)

Figure 11: Control action after the critical time instant $t_{c}=43.6 \mathrm{~s}$; MCSI (black line), MCSI-PP (red line) and $\sigma$-MCSI (blue line): (a) $t \in[43,44.5] \mathrm{s}$; (b) $t \in[51.08,51.13] \mathrm{s}$.

We finally point out that the residual tracking error detected for the MCSI-PP and $\sigma$-MCSI in Figures 8 and 10 is due to the disturbance in (25). As the frequency of this disturbance can be estimated, its effect on the closed-loop dynamics could be further mitigated by using additional adaptive control terms such as those presented in [36]. Nevertheless, we again recall that the aim of this work is to show the effectiveness of the gain-locking solutions proposed in [26] and summarized in 
Section 2 to prevent instability in the adaptive gains of MCSI control algorithms. Hence, the adaptive rejection of periodic disturbances is out of the scope of this study.

\section{Conclusions}

In this article we have experimentally validated systematic solutions, which have been recently proposed in the technical literature, to prevent unbounded diverging control gains that may arise when minimal control synthesis adaptive algorithms are used to control systems affected by unmodelled nonlinear dynamics, unknown parameters and disturbances.

The selected experimental plant was an electro-mechanical system subject to significant, rapidly varying persistent disturbances, and parametric uncertainty. Experimental results confirmed the effectiveness of the MCSI-based methods to keep the integral part of the adaptive gains of the algorithm in a preassigned set without compromising the tracking of a reference trajectory.

\section{Acknowledgments}

R. Costa-Castelló was partially supported by the Spanish National Project MICINN DPI2015-69286-C3-2-R MINECO/FEDER. J.M. Olm was partially supported by the Government of Spain through the Agencia Estatal de Investigacion Project DPI2017-85404-P and the Generalitat de Catalunya Project 2017 SGR 872.

\section{References}

[1] B.D.O. Anderson, R.R. Bitmead, C.R. Johnson, P.V. Kokotovic, R.L. Kosut, I.M.Y. Mareels, L. Praly, and B.D. Riedle. Stability of adaptive systems: passivity and averaging analysis. The M.I.T. Press, Cambridge, MA and London, 1986.

[2] R. Ashok and Y. Shtessel. Control of fuel cell-based electric power system using adaptive sliding mode control and observation techniques. J. Franklin Institute, 352(11):4911-4934, 2015.

[3] C. Larco Barros, J. M. Olm, and R. Costa-Castellló. Rejection of periodic disturbances using MRAC with minimal controller synthesis. In Proc. 21st IEEE International Conference on Emerging Technologies and Factory Automation, 2016. 
[4] H. Benchoubane and D.P. Stoten. The decentralized minimal controller synthesis algorithm. Internat. J. Control, 56(4):967-983, 1992.

[5] O.S. Bursi, D.P. Stoten, and L. Vulcan. Convergence and frequency-domain analysis of a discrete first-order model reference adaptive controller. Structural Control and Health Monitoring, 14(5):777-807, 2007.

[6] R. Chi, Z. Hou, and S. Jin. A data-driven adaptive ILC for a class of nonlinear discrete-time systems with random initial states and iteration-varying target trajectory. J. Franklin Institute, 352(6):2407-2424, 2015.

[7] R. Costa-Castelló, J. Nebot, and R. Griñó. Demonstration of the internal model principle by digital repetitive control of an educational laboratory plant. IEEE Trans. Education, 48(1):73-80, 2005.

[8] M. di Bernardo, A. di Gaeta, U. Montanaro, J. M. Olm, and S. Santini. Experimental validation of the discrete-time MCS adaptive strategy. Control Engineering Practice, 21(6):847-859, 2013.

[9] M. di Bernardo, F. di Gennaro, J. M. Olm, and S. Santini. Discrete-time minimal control synthesis adaptive algorithm. Internat. J. Control, 83(4):26412657, 2010.

[10] M. di Bernardo, C.I. Hoyos-Velasco, U. Montanaro, and S. Santini. Experimental implementation and validation of a novel minimal control synthesis adaptive controller for continuous bimodal piecewise affine systems. Control Engineering Practice, 20(3):269-281, Mar. 2012.

[11] M. di Bernardo, U. Montanaro, J.M. Olm, and S. Santini. Model reference adaptive control of discrete-time piecewise linear systems. International Journal of Robust and Nonlinear Control, 23(7):709-730, 2013.

[12] M. di Bernardo, U. Montanaro, R. Ortega, and S. Santini. Extended hybrid model reference adaptive control of piecewise affine systems. Nonlinear Analysis: Hybrid Systems, 21:11-21, August 2016.

[13] M. di Bernardo, U. Montanaro, and S. Santini. Hybrid model reference adaptive control of piecewise affine systems. IEEE Transactions on Automatic Control, 58(2):304-316, 2013. 
[14] A. di Gaeta, C.I. Hoyos-Velasco, and U. Montanaro. Cycle-by-cycle adaptive force compensation for the soft-landing control of an electro-mechanical engine valve actuator. Asian Journal of Control, 17(5):1707-1724, 2015.

[15] G. Fedele and A. Ferrise. Periodic disturbance rejection with unknown frequency and unknown plant structure. J. Franklin Institute, 351(2):10741092, 2014.

[16] W. He, Y. Chen, and Z. Yin. Adaptive neural network control of an uncertain robot with full-state constraints. IEEE Trans. Cybernetics, 46(3):620-629, 2016.

[17] W. He, Y. Dong, and C. Sun. Adaptive neural impedance control of a robotic manipulator with input saturation. IEEE Trans. Systems, Man, and Cybernetics: Systems, 46(3):334-344, 2016.

[18] W. He and S. Sam Ge. Cooperative control of a nonuniform gantry crane with constrained tension. Automatica, 66(4):146-154, 2016.

[19] W. He, Z. Yan, C. Sun, and Y. Chen. Adaptive neural network control of a flapping wing micro aerial vehicle with disturbance observer. IEEE Trans. Cybernetics, 47(10):3452-3465, 2017.

[20] W. He and S. Zhang. Control design for nonlinear flexible wings of a robotic aircraft. IEEE Trans. Control Systems Technology, 25(1):351-357, 2017.

[21] H.K. Khalil. Nonlinear Systems. Prentice Hall, third edition, 2002.

[22] Y. D. Landau. Adaptive Control: The Model Reference Approach. CRC Press, 1979.

[23] U. Montanaro, A. di Gaeta, and V. Giglio. Adaptive tracking control of a common rail injection system for gasoline engines: a discrete-time integral minimal control synthesis approach. IEEE Transactions on Control Systems Technology, 21(5):1940-1948, 2013.

[24] U. Montanaro, A. di Gaeta, and V. Giglio. Robust discrete-time MRAC with minimal controller synthesis of an electronic throttle body. IEEE/ASME Transactions on Mechatronics, 19(2):524 - 537, 2014. 
[25] U. Montanaro and J. M. Olm. Discrete-time integral MRAC with minimal controller synthesis and parameter projection. Journal of The Franklin Institute, 352(12):5415-5436, 2015.

[26] U. Montanaro and J. M. Olm. Integral MRAC with minimal controller synthesis and bounded adaptive gains: the continuous-time case. Journal of Franklin Institute, 353(18):5040-5067, 2016.

[27] Y. Pan and G-H. Yang. Event-triggered fuzzy control for nonlinear networked control systems. Fuzzy Sets and Systems, 329(C):91-107, 2017.

[28] D.P. Stoten. The adaptive minimal control synthesis algorithm with integral action. In Proc. 21st Internat. Conf. Industrial Electronics, Control, and Instrumentation, volume 2, pages 1646-1651, 1995.

[29] D.P. Stoten and H. Benchoubane. Empirical studies of an MRAC algorithm with minimal controller synthesis. Internat. J. Control, 51(4):823-849, 1990.

[30] D.P. Stoten and H. Benchoubane. Robustness of minimal controller synthesis algorithm. Internat. J. Control, 51(4):851-861, 1990.

[31] D.P. Stoten and H. Benchoubane. The extended minimal controller sysnthesis algorithm. Internat. J. Control, 56(5):1139 - 1165, 1992.

[32] D.P. Stoten and M. di Bernardo. Application of the minimal control synthesis algorithm to the control and synchronization of chaotic systems. Internat. J. Control, 65(6):925-938, 1996.

[33] D.P. Stoten and E.G. Gómez. Adaptive control of shaking tables using the minimal controller synthesis algorithm. Philosophical Trans. Royal Soc. London, 357(9):1697-1723, 2001.

[34] D.P. Stoten and S.A. Neild. The error-based minimal control synthesis algorithm with integral action. Proc. Inst. of Mechanical Eng. - Part I: J. Systems and Control Eng., 217(3):187-201, 2003.

[35] R. Ganapathy Subramanian, V. Kumar Elumalai, S. Karuppusamy, and V. Krishna Canchi. Uniform ultimate bounded robust model reference adaptive pid control scheme for visual servoing. J. Franklin Institute, 354(4):1741-1758, 2017. 
[36] G. Tao. Adaptive Control Design and Analysis. John Wiley \& Sons, Inc. Hoboken, NJ, 2003.

[37] L-B. Wu, X-Q. He, and D-Q. Zhang. Cooperative adaptive fuzzy control for a class of uncertain non-linear multi-agent systems with time delays. Journal of Control and Decision, 4(3):131-152, 2017.

[38] Y. Yang, X. Chen, and C. Li. Transient performance improvement in model reference adaptive control using image optimal method. J. Franklin Institute, 352(1):16-32, 2015.

[39] Y. Yang, C. Ge, H. Wang, X. Li, and C. Hua. Adaptive neural network based prescribed performance control for teleoperation system under input saturation. J. Franklin Institute, 352(5):1850-1866, 2015. 